\title{
Bimaxillary Osteotomy Using a Physiological Positioning Strategy for Skeletal Class II with Anterior Open Bite and Gummy Smile: Case Reports and Review of the Literature
}

\author{
Seigo Ohba ${ }^{1,2 *}$, Hitoshi Yoshimura², Takiko Matsuura ${ }^{2}$, Izumi Asahina ${ }^{1}$ and Kazuo Sano ${ }^{2}$ \\ ${ }^{1}$ Department of Regenerative Oral Surgery, Graduate School of Biomedical Sciences, Nagasaki University, Nagasaki, Japan \\ ${ }^{2}$ Division of Dentistry and Oral Surgery, Department of Sensory and Locomotor Medicine, Faculty of Medical Sciences, University of Fukui, Fukui, Japan
}

\begin{abstract}
The treatment of skeletal class II cases has long been a great challenge because of the tendency for relapse. This is particularly true in skeletal class II patient with an anterior open bite and gummy smile, owing to the anterior movement and counter-clockwise rotation of the mandible. This movement of the mandible sometimes induces relapse and temporomandibular disorders. To avoid these complications, we have advocated a new treatment strategy, the 'physiological positioning strategy', for mandibular osteotomy in patients with jaw deformity. Few disadvantages were observed after surgery when using this strategy, and good outcomes were seen in skeletal class III patients who underwent bimaxillary osteotomy or mandibular osteotomy solely. Two skeletal class II patients with anterior open bite and a gummy smile were treated using this method. The long-term outcomes were good in both cases. The proximal segments moved anteriorly after surgery. This new treatment strategy may provide a reliable outcome for not only class III but also class II cases, even when concomitant anterior open bite and a gummy smile are present.
\end{abstract}

Keywords: Physiological positioning strategy; Skeletal class II; Gummy smile; Open bite

\section{Introduction}

The treatment of anterior open bite (AOB) in a non-growing patient has long been a great challenge because of the tendency for relapse (25$38 \%$ incidence) [1]. There are a number of treatment strategies available to treat jaw deformities with $\mathrm{AOB}$, such as orthodontic therapy, maxillary osteotomy, mandibular osteotomy, or bimaxillary osteotomy [2-5]. However, relapse tends to occur after orthodontic therapy and maxillary osteotomy $[2,3]$. Moreover, mandibular osteotomy with counter-clockwise rotation has shown extensive relapse in severe AOB cases. Therefore, the application of mandibular osteotomy has been limited to patients with mild-to-moderate AOB [4]. Although there has been a report that bimaxillary osteotomy provided a good treatment outcome in a patient with $\mathrm{AOB}$, relapse of at least $2 \mathrm{~mm}$ has been reported to occur after bimaxillary osteotomy to treat $\mathrm{AOB}[5,6]$. It has also been suggested that the stability following bimaxillary osteotomy is similar to that after maxillary osteotomy to treat AOB. According to the above previous reports, there does not yet appear to be a consensus and/or reliable treatment strategy.

Gummy smile, referring to the excessive gingival exposure in the anterior maxilla during smiling, is another challenging treatment. In the case of a gummy smile, the maxillary segment must be impacted and/or rotated in a counter-clockwise direction after maxillary osteotomy. It is known that the skeletal stability of the segment undergoing impaction and/or counter-clockwise rotation is reliable following maxillary osteotomy because of sufficient bone contact between the segments after movement [7]. However, movement of this maxillary segment induces a further degree of counter-clockwise rotation of the mandible, aside from the skeletal instability of the mandible following osteotomy with counter-clockwise rotation. Therefore, it may be difficult to gain adequate skeletal stability post-surgery in cases with a gummy smile.

The movement of the mandible anteriorly after surgery is unreliable; similar to mandibular counter-clockwise rotation [8]. Cases where the segment needs to be moved anteriorly after mandibular osteotomy are usually class II skeletal patterns. Patients with skeletal class II often suffer from temporomandibular joint disorder (TMD)
[9]. Moreover, anterior movement of the segment often induces TMD after mandibular osteotomy [10]. Additionally, progressive condylar resorption (PCR), which has been a problem after sagittal split ramus osteotomy (SSRO) for a decade, needs to be considered, even if the details of its developmental mechanism remain unknown [11]. The risks of relapse and the development of TMD, including PCR, have been implicated after orthognathic surgery in skeletal class II patients. Therefore, achieving a good treatment outcome in a skeletal class II patient with $\mathrm{AOB}$ and a gummy smile is expected to be quite difficult.

It is still unknown whether the original ramus position is physiologically ideal for a newly created occlusion and jaw movement after orthognathic surgery [12]. Nonetheless, attempts have been made to return the proximal segments to their original position and some devices were reported to have achieved this objective [13]. However, there were some reports that TMD and PCR occurred after SSRO with fixation of the segments $[10,11]$. As mentioned above, the position of the proximal segments after surgery remains controversial. We hypothesized that the original position of the ramus is not always physiologically ideal for a newly created occlusion and for jaw movement after orthognathic surgery. To induce the proximal segments to the physiologically ideal position and to avoid the aforementioned postsurgical complications, we created a unique treatment strategy, the Physiological Positioning Strategy (PPS) [14,15]. Using this method, a

*Corresponding author: Seigo Ohba, DDS, PhD, FIBCSOMS, Department of Regenerative Oral Surgery, Unit of Translational Medicine, Graduate School of Biochemical Science, Nagasaki University, 1-7-1 Sakamoto, Nagasaki 852-8588, Japan, Tel: 81 958197704; Fax: 81 958197705; E-mail: seigoohba@gmail.com/ sohba@nagasaki-u.ac.jp

Received December 15, 2015; Accepted January 07, 2015; Published January 14, 2016

Citation: Ohba S, Yoshimura H, Matsuura T, Asahina I, Sano K (2016) Bimaxillary Osteotomy Using a Physiological Positioning Strategy for Skeletal Class II with Anterior Open Bite and Gummy Smile: Case Reports and Review of the Literature. Dentistry 6: 356. doi:10.4172/2161-1122.1000356

Copyright: $\odot 2016$ Ohba S, et al. This is an open-access article distributed under the terms of the Creative Commons Attribution License, which permits unrestricted use, distribution, and reproduction in any medium, provided the original author and source are credited. 
Citation: Ohba S, Yoshimura H, Matsuura T, Asahina I, Sano K (2016) Bimaxillary Osteotomy Using a Physiological Positioning Strategy for Skeletal Class II with Anterior Open Bite and Gummy Smile: Case Reports and Review of the Literature. Dentistry 6: 356. doi:10.4172/21611122.1000356

short lingual osteotomy (SLO), which is a modification of the SSRO, is performed for the mandible and the segment is not fixed. Subsequently, jaw exercise with elastics is initiated on the second day after surgery. This postoperative management is the same as our previous report for intraoral vertical ramus osteotomy (IVRO) [12]. This new treatment strategy provided good outcomes after mandibular or bimaxillary osteotomies for skeletal class III cases [14,15].

Here, we report two cases of skeletal class II with $\mathrm{AOB}$ and a gummy smile. They underwent bimaxillary osteotomies and the maxillary segments were moved upward with counter-clockwise rotation. Subsequently, the mandibles were moved anteriorly with counter-clockwise rotation. They were followed with PPS after surgery (Figures 1-7).

\section{Case Reports}

\section{Case-1}

Diagnosis and etiology: A 25 year old Japanese woman was referred to the Department of Dentistry and Oral Surgery, University of Fukui Hospital because of an $\mathrm{AOB}$ and excessive gingival exposure in the anterior maxilla during smiling. An overbite of $-2.0 \mathrm{~mm}$ and an overjet of $3.0 \mathrm{~mm}$ were noted. During routine examination, she was diagnosed with skeletal class II with an AOB and gummy smile (Figure 1 ). The data from cephalometric analyses of before (T1), immediately after (T2), 3 months after (T3), 6 months after (T4), and 12 months after surgery (T5) are shown in Table 1 . There were no symptoms of TMD before surgery. She underwent bimaxillary osteotomies to correct her skeletal class II with AOB and gummy smile.

Treatment objectives: According to the previous reports, the combination of Le Fort I and horseshoe osteotomies for the maxilla were performed $[16,17]$. The maxillary segment was down fractured after conventional Le Fort I osteotomy. Then the alveolo-palatal bone osteotomy of the segment was performed by using PiezoSurgery ${ }^{\circledR}$ (Mectron medical technology, Carasco, Italy) for horseshoe osteotomy without disturbance of the palatal mucosa and the descending palatine artery. The dentoalveolar segment was moved 6.5 and $4.5 \mathrm{~mm}$ upwards at the anterior and posterior regions, respectively. The segment was fixed at the lateral side of the nasal aperture by $0.7 \mathrm{~mm}$ titanium plates

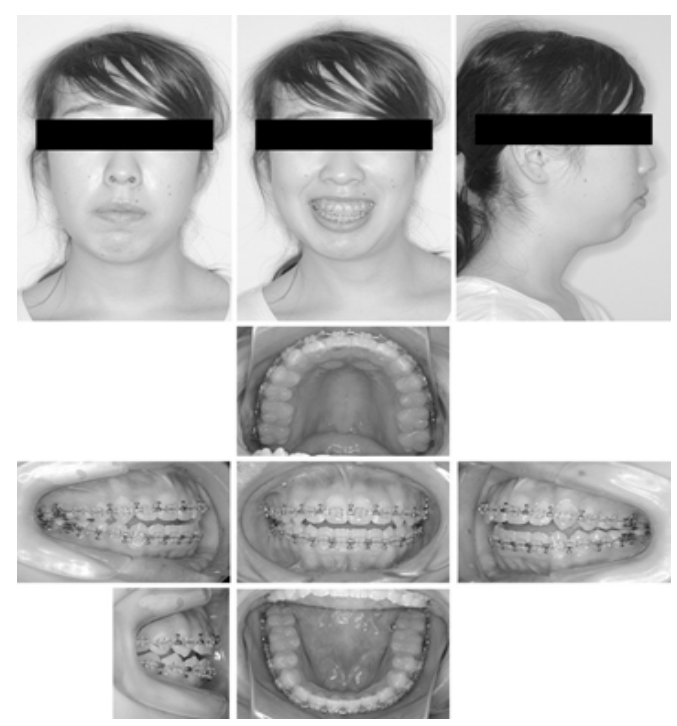

Figure 1: Facial and intraoral appearance before surgery of Case-1.
(Matrix Midface, Depuy Synthes, Oberdorf, Switzerland) and at the root of the zygoma by $0.8 \mathrm{~mm}$ titanium plates on each side. Each plate was fixed rigidly with four screws. Subsequently, SLO was performed for the mandible without segmental fixation. A surgical acrylic splint was worn in the upper dental arch. Finally, inter-maxillary fixation (IMF) was performed with $0.3 \mathrm{~mm}$ stainless steel wires, which were hooked on IMF screws (JEIL Dual-Top Auto Screw, Proceed, Korea) on the jaw bones.

\section{Case-2}

Diagnosis and etiology: An 18 year old Japanese woman was referred to the Department of Dentistry and Oral Surgery, University of Fukui Hospital. Her chief complaint was mandibular retrognathia. $\mathrm{AOB}$ and excessive gingival exposure in the anterior maxilla during smiling were also observed (Figure 4). An overbite of $-1.5 \mathrm{~mm}$ and an overjet of $5.5 \mathrm{~mm}$ were noted. The data from cephalometric analyses of $\mathrm{T} 1-\mathrm{T} 5$ are shown in Table 2 . There were no symptoms of TMD before surgery.

Treatment objectives: She underwent bimaxillary osteotomies for her skeletal class II with AOB and gummy smile. A conventional Le Fort I osteotomy was performed for the maxilla and the segment was moved upwards $4.5 \mathrm{~mm}$ in the anterior region, $2.5 \mathrm{~mm}$ in the right molar region, and $3.5 \mathrm{~mm}$ in the left molar region. Finally, the segment was moved posteriorly by $3.0 \mathrm{~mm}$. The segment was fixed at the lateral side of the nasal aperture with $0.7 \mathrm{~mm}$ titanium plates (Matrix Midface, Depuy Synthes, Oberdorf, Switzerland) and at the root of the zygoma by $0.8 \mathrm{~mm}$ titanium plates on each side. Each plate was fixed rigidly with four screws. Subsequently, a SLO was performed for the mandible without segmental fixation. A surgical acrylic splint was worn in the upper dental arch. Finally, IMF was performed with $0.3 \mathrm{~mm}$ stainless wires, which were hooked on IMF screws (JEIL Dual-Top Auto Screw) on the jaw bones.

\section{Treatment progress}

The postoperative management in both cases was followed by the PPS regimen $[14,15]$. Jaw exercise was initiated on the second day after surgery while wearing the surgical acrylic splint and elastics instead of IMF wires. To inhibit the eruption force subjected to teeth, elastics were hooked on IMF screws during jaw exercise. Postsurgical orthodontic treatment was initiated in the seventh week after surgery in both cases. IMF screws were removed 5 months after surgery in Case- 1 . In Case2 , the screws were removed 10 weeks after surgery because of their loosening. Facial and intraoral appearances before and 12 months after surgeries are shown in Figure 2 for Case- 1 and Figure 5 for Case-2. In both cases, no excessive gingival exposure in the anterior maxilla was observed. Jaw opening width was recovered 6 months after surgery to approximately the same degree as it was originally (Figure 7). No TMD symptoms were observed in either case after surgery. No sensory disability of the inferior alveolar nerve was observed in either case 3 months after surgery. Finally, the ideal overjet $(2.0 \mathrm{~mm})$ and overbite $(3.0 \mathrm{~mm})$ were acquired in both cases 12 months after surgery. The lateral profiles were superimposed in Figure 3 for Case- 1 and Figure 6 for Case-2.

\section{Results and Cephalometric Analysis}

The skeletal profile of $\mathrm{T} 2$ was almost within the average range for a Japanese woman in Case-1 (Table 1). The mandibular plane and occlusal plane angles decreased by $12.1^{\circ}$ and $10.4^{\circ}$ after surgery, respectively. This implied counter-clockwise rotation of the maxilla- 
Citation: Ohba S, Yoshimura H, Matsuura T, Asahina I, Sano K (2016) Bimaxillary Osteotomy Using a Physiological Positioning Strategy for Skeletal Class II with Anterior Open Bite and Gummy Smile: Case Reports and Review of the Literature. Dentistry 6: 356. doi:10.4172/21611122.1000356

Page 3 of 6

\begin{tabular}{|c|c|c|c|c|c|c|}
\hline Parameter & Standard & $\mathrm{T1}$ & T2 & T3 & T4 & T5 \\
\hline Facial angle & $84.83 \pm 3.05$ & $79.5^{*}$ & 87.9 & 87.7 & 86.8 & 86.5 \\
\hline Convexity & $7.58 \pm 4.95$ & $15.5^{*}$ & 8.7 & 9.5 & $13.6^{*}$ & 12.3 \\
\hline A-B plane & $-4.48 \pm 3.50$ & $-9.1^{*}$ & -6.0 & -7.2 & $-8.9^{*}$ & $-8.3^{*}$ \\
\hline Mandibular plane & $28.81 \pm 5.23$ & $44.6^{*}$ & 32.5 & 33.2 & $35.5^{*}$ & $35.1^{*}$ \\
\hline Y-axis & $65.38 \pm 5.63$ & $73.2^{*}$ & 63.2 & 64.1 & 65.4 & 65.0 \\
\hline Occlusal plane & $11.42 \pm 3.64$ & $20.9^{*}$ & 10.5 & 9.9 & 10.6 & 10.9 \\
\hline Interincisal & $124.09 \pm 7.63$ & $116.3^{*}$ & 121.1 & 120.4 & 121.8 & 119.3 \\
\hline L-1 to occlusal plane & $23.84 \pm 5.28$ & $29.6^{*}$ & 27.9 & 28.9 & $30.1^{*}$ & $31.8^{*}$ \\
\hline $\begin{array}{l}\mathrm{L}-1 \text { to mandibular } \\
\text { plane }\end{array}$ & $96.33 \pm 5.78$ & 95.9 & 95.9 & 95.6 & 95.2 & 97.5 \\
\hline $\mathrm{FH}$ to $\mathrm{SN}$ & $6.19 \pm 2.89$ & $9.5^{*}$ & $9.7^{*}$ & $9.7^{*}$ & $9.5^{*}$ & $9.9^{*}$ \\
\hline SNA & $82.32 \pm 3.45$ & $78.2^{*}$ & 83.0 & 83.3 & 84.8 & 83.3 \\
\hline SNB & $78.90 \pm 3.45$ & $70.5^{\star}$ & 77.9 & 77.2 & 76.9 & 76.2 \\
\hline SNA-SNB deff. & $3.39 \pm 1.77$ & $7.8^{*}$ & 5.2 & 6.1 & $7.9^{*}$ & $7.2^{*}$ \\
\hline $\mathrm{U}-1$ to $\mathrm{FH}$ plane & $111.13 \pm 5.54$ & $103.2^{*}$ & 110.5 & 110.8 & 107.5 & 108.0 \\
\hline $\mathrm{U}-1$ to $\mathrm{SN}$ plane & $104.54 \pm 5.55$ & $93.7^{\star}$ & 100.9 & 101.2 & $98.0^{*}$ & $98.1^{*}$ \\
\hline Gonial angle & $122.23 \pm 4.61$ & $133.5^{*}$ & $131.5^{*}$ & $132.4^{*}$ & $134.5^{*}$ & $134.0^{*}$ \\
\hline Ramus inclination & $2.93 \pm 4.40$ & -1.0 & $9.0^{*}$ & $9.2^{*}$ & $9.0^{*}$ & $8.9^{*}$ \\
\hline FMIA & $54.6 \pm 6.5$ & $39.5^{*}$ & 51.6 & 51.2 & 49.3 & $47.4^{*}$ \\
\hline
\end{tabular}

Table 1: Cephalometric analysis of Case-1.

\begin{tabular}{|c|c|c|c|c|c|c|}
\hline Parameter & Standard & T1 & T2 & T3 & T4 & T5 \\
\hline Facial angle & $84.83 \pm 3.05$ & 82.5 & 87.3 & 84.1 & 84.1 & 83.6 \\
\hline Convexity & $7.58 \pm 4.95$ & $16.4^{*}$ & 4.4 & 12.1 & 12.4 & $13.3^{*}$ \\
\hline A-B plane & $-4.48 \pm 3.50$ & $-9.3^{*}$ & -3.7 & -7.1 & -7.7 & $-8.7^{\star}$ \\
\hline Mandibular plane & $28.81 \pm 5.23$ & 32.9 & 26.3 & 29.8 & 29.6 & 30.0 \\
\hline Y-axis & $65.38 \pm 5.63$ & 66.3 & 61.3 & 64.1 & 64.2 & 65.0 \\
\hline Occlusal plane & $11.42 \pm 3.64$ & $15.2^{*}$ & $6.2^{*}$ & 9.0 & 9.1 & 10.3 \\
\hline Interincisal & $124.09 \pm 7.63$ & 118.4 & 120.3 & 122.5 & 124.6 & 125.2 \\
\hline L-1 to occlusal plane & $23.84 \pm 5.28$ & 28.8 & $29.3^{*}$ & $30.9^{*}$ & $29.4^{*}$ & $30.4^{*}$ \\
\hline $\begin{array}{l}\mathrm{L}-1 \text { to mandibular } \\
\text { plane }\end{array}$ & $96.33 \pm 5.78$ & 101.0 & 99.2 & 100.1 & 98.9 & 100.7 \\
\hline $\mathrm{FH}$ to $\mathrm{SN}$ & $6.19 \pm 2.89$ & 8.6 & 8.6 & 8.6 & 8.6 & 8.8 \\
\hline SNA & $82.32 \pm 3.45$ & 82.6 & 81.1 & 82.0 & 82.1 & 81.9 \\
\hline SNB & $78.90 \pm 3.45$ & $74.2^{*}$ & 78.1 & 75.6 & 75.5 & $74.7^{*}$ \\
\hline SNA-SNB deff. & $3.39 \pm 1.77$ & $8.4^{*}$ & 3.0 & $6.4^{*}$ & $6.6^{*}$ & $7.1^{*}$ \\
\hline U-1 to $\mathrm{FH}$ plane & $111.13 \pm 5.54$ & 107.6 & 114.2 & 107.7 & 106.9 & $104.1^{*}$ \\
\hline U-1 to SN plane & $104.54 \pm 5.55$ & 99.0 & 105.5 & 99.1 & $98.2^{*}$ & $95.4^{*}$ \\
\hline Gonial angle & $122.23 \pm 4.61$ & 120.3 & 122.1 & 126.8 & 126.7 & 126.1 \\
\hline Ramus inclination & $2.93 \pm 4.40$ & $-2.7^{*}$ & 5.7 & 7.0 & 7.1 & 6.1 \\
\hline FMIA & $54.6 \pm 6.5$ & $46.0^{*}$ & 54.4 & 50.1 & 51.5 & 49.3 \\
\hline
\end{tabular}

Table 2: Cephalometric analysis of Case-2.

mandibular bone segment. Moreover, the facial angle and SNB increased by $8.4^{\circ}$ and $7.4^{\circ}$, respectively, and Y-axis decreased by $10.0^{\circ}$ after surgery. These changes indicated anterior-superior movement of the mandible. The cephalometric values of almost all measured angles from T3 to T5 showed few changes. However, the changes in facial angle, $\mathrm{SNB}$, and inter-incisal angle from $\mathrm{T} 2$ to $\mathrm{T} 5$ were $-1.4^{\circ},-1.7^{\circ}$, and $-1.8^{\circ}$, respectively, and the changes from $\mathrm{T} 2$ to $\mathrm{T} 5$ in the mandibular plane angle and Y-axis were $2.6^{\circ}$ and $1.8^{\circ}$, respectively. There was no change in SNA $\left(0.3^{\circ}\right)$ and occlusal plane angle $\left(0.4^{\circ}\right)$ from T2 to T5. This indicated that the skeletal stability of the maxilla was excellent and that of the mandible was slightly poorer but acceptable 1 year postsurgery. This small relapse could be recovered by the linguoclination of the upper anterior teeth and the labioclination of the lower anterior teeth. Interestingly, the ramal inclination was $-1.0^{\circ}$ at $\mathrm{T} 1$ and the ramus swung after surgery. Finally, the inclination of the ramus was $8.9^{\circ}$ at $\mathrm{T} 5$. The ramus swung anteriorly by $9.9^{\circ}$.

Regarding Case-2 (Table 2), the mandibular plane and occlusal plane angles decreased by $6.6^{\circ}$ and $9.0^{\circ}$ after surgery, respectively. This implied counter-clockwise rotation of the maxilla-mandibular bone segment. Moreover, the facial angle and SNB increased by $4.8^{\circ}$ and $3.9^{\circ}$, respectively, and $\mathrm{Y}$-axis decreased by $5.0^{\circ}$ after surgery. These outcomes implied anterior-superior movement of the mandible. Almost all measured angles showed few changes from T3 to T5. From T2 to T5, the changes in facial angle, mandibular plane angle and $\mathrm{Y}$-axis were $3.7^{\circ}$ each, and in SNB and inter-incisal angle, the changes were $4.1^{\circ}$ and $4.9^{\circ}$, respectively. The change in both U1-SN and U1-FH from $\mathrm{T} 2$ to $\mathrm{T} 5$ was approximately $-10^{\circ}$. The change in SNA during the same interval was $0.8^{\circ}$. These movements showed that the maxillary segment did not change in the anterior-posterior direction but the maxilla-mandibular segment rotated in a counter-clockwise direction. The skeletal relapse was compensated for by the inclination and slight eruption of the upper anterior teeth. Interestingly, the ramal inclination was $-2.7^{\circ}$ at $\mathrm{T} 1$, and the ramus swung after surgery in a similar manner to Case-1. Finally, the ramal inclination at T5 was $6.1^{\circ}$. The ramus swung anteriorly by $8.8^{\circ}$. 
Citation: Ohba S, Yoshimura H, Matsuura T, Asahina I, Sano K (2016) Bimaxillary Osteotomy Using a Physiological Positioning Strategy for Skeletal Class II with Anterior Open Bite and Gummy Smile: Case Reports and Review of the Literature. Dentistry 6: 356. doi:10.4172/21611122.1000356

Page 4 of 6

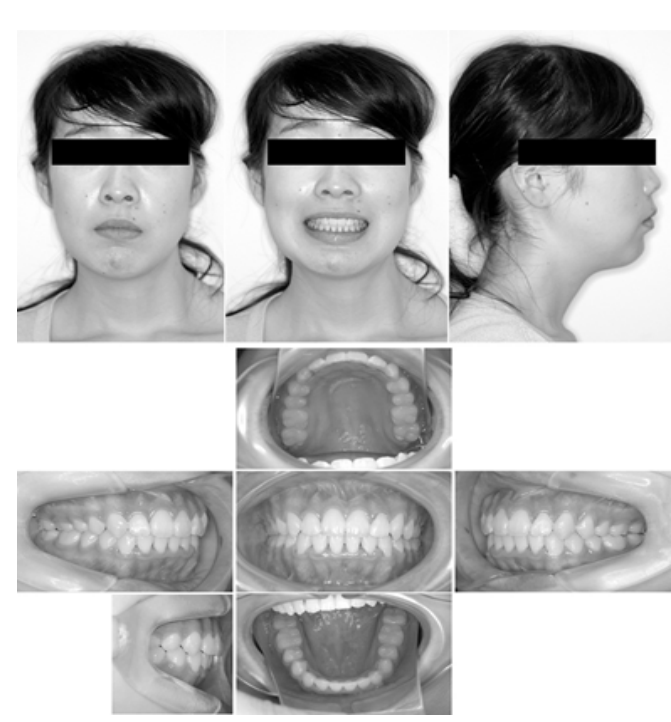

Figure 2: Facial and intraoral appearance 12 months after surgery of Case-1.

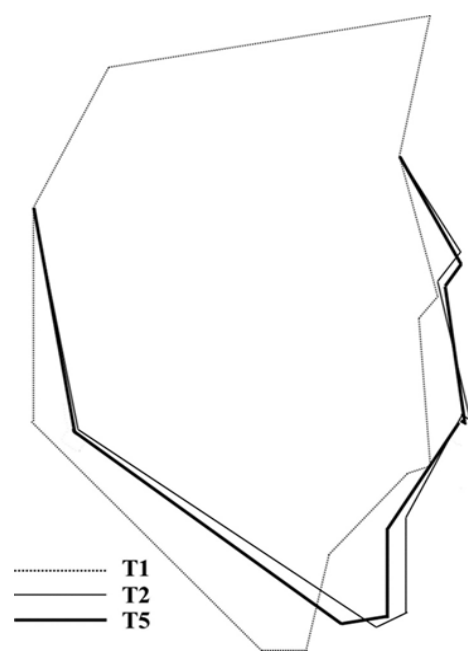

Dotted line; $\mathrm{T} 1$; before surgery, Thin line; T2; immediately after surgery, Thick line; T5; 12 months after surgery.

Figure 3: Superimposed cephalometric illustrations of Case-1.

\section{Discussion}

To move the mandible with counter-clockwise rotation using orthodontic treatment to treat the $\mathrm{AOB}$, reduction of the posterior teeth and traction by elastics is necessary. Inappropriate forces applied to teeth often results in adverse effects, such as alveolar bone dehiscence and tooth root resorption $[18,19]$. Orthodontic treatment strategies with skeletal anchorage devices (SADs) have been used to avoid these complications. One of the most crucial advantages of SADs such as miniscrews and miniplates was reported to be the minimal invasiveness of the procedure. The success rates of miniscrews and miniplates have been reported to be $91.4-100 \%$ and $61-100 \%$, respectively [20]. Because wide ranges of success rates have been reported, especially with miniplates, it is not certain that these devices are completely reliable. Moreover, plate bending and the surgical treatment necessary for setting some miniplates are often complicated because of the complexity of the bone surface. Therefore, it is hard to say whether SADs are always minimally invasive. Regarding miniscrews, their application involves a risk for tooth root damage [21]. Kaku et al. reported a case of a skeletal class II patient with a gummy smile who underwent orthodontic treatment with SADs [22]. Nishimura et al. also reported a case of a skeletal class II, division 2 patient who underwent orthodontic treatment with SADs. Both reports showed good outcomes [23]. However, Capelozza et al. concluded that it was difficult to treat a dolichofacial patient by orthodontic treatment with SADs and the surgical approach provided a good outcome [24]. This indicates possible limitations of orthodontic treatment using SADs.

Ribeiro et al. reported a case of a 14 year old girl with an $\mathrm{AOB}$ who was treated by orthodontics using a chin-cup, resulting in a good outcome [25]. The fact that she was in the developmental stage may be one of the crucial factors to obtaining a good outcome. In the present two cases, both patients had completed their growth. Therefore, it was

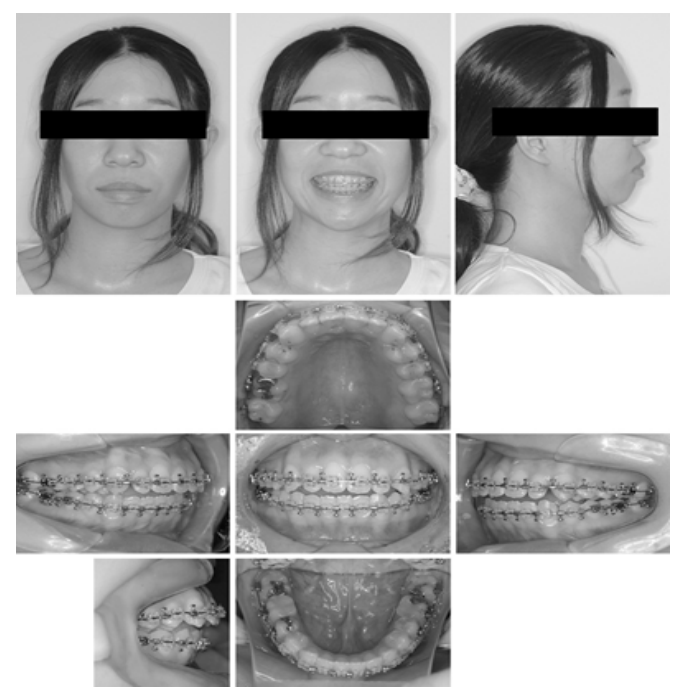

Figure 4: Facial and intraoral appearance before surgery of Case-2.

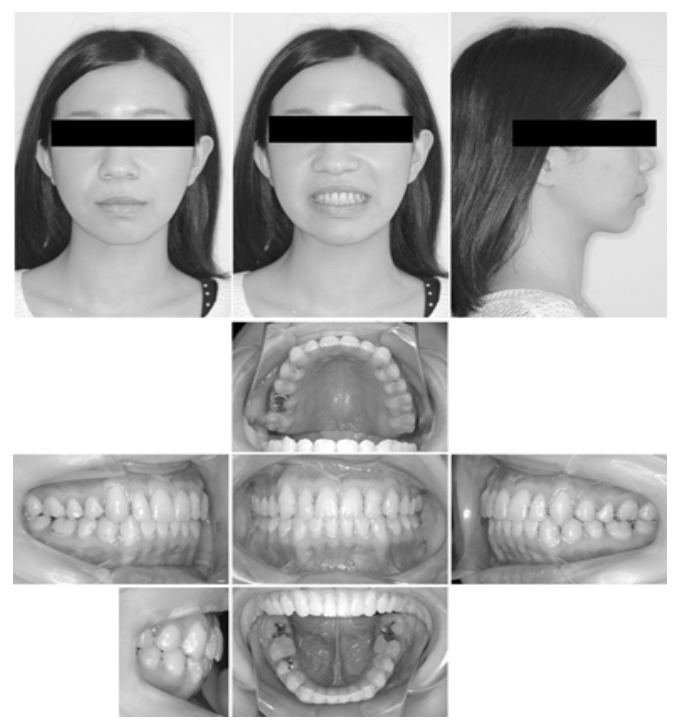

Figure 5: Facial and intraoral appearance 12 months after surgery of Case-2. 
Citation: Ohba S, Yoshimura H, Matsuura T, Asahina I, Sano K (2016) Bimaxillary Osteotomy Using a Physiological Positioning Strategy for Skeletal Class II with Anterior Open Bite and Gummy Smile: Case Reports and Review of the Literature. Dentistry 6: 356. doi:10.4172/21611122.1000356

Page 5 of 6

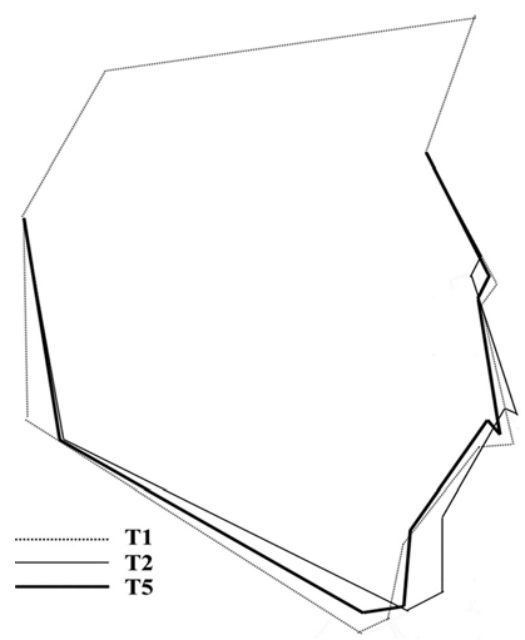

Dotted line; T1; before surgery, Thin line; T2; immediately after surgery, Thick line; T5; 12 months after surgery.

Figure 6: Superimposed cephalometric illustrations of Case-2.

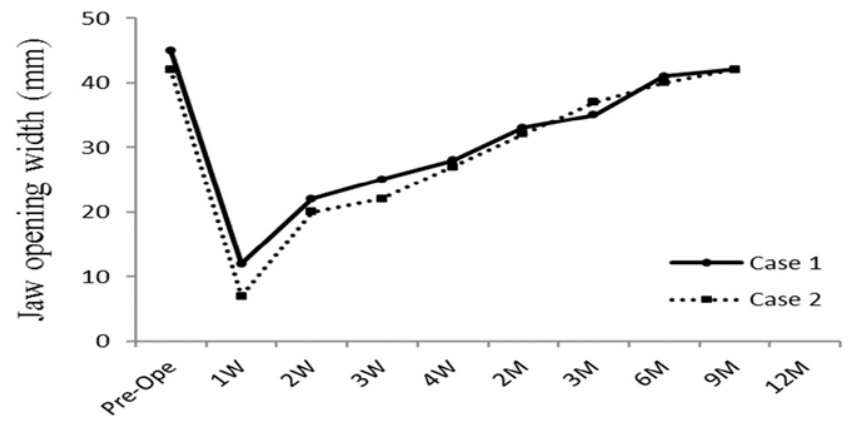

Pre-Ope; before surgery, W; week(s), M; months

Figure 7: The change in mouth opening width after surgery.

impossible to control the growth of their jaws in treating their AOBs. Moreover, both patients were diagnosed with skeletal class II. This suggested that the mandible needed to move anteriorly. According to these diagnoses, the patients underwent both orthodontic and orthognathic surgical treatments.

Shimo et al. reported that the combination of Le Fort I and horseshoe osteotomies for the maxilla and an IVRO for the mandible were performed in a patient with severe gummy smile, resulting in a good treatment outcome [26]. According to this report, the combination of Le Fort I and horseshoe osteotomies for the maxilla was performed in Case-1. Regarding the mandible, it was necessary to move the mandible forward in the present case, as the patient was diagnosed with skeletal class II with concomitant AOB and a gummy smile. This is why SSRO was chosen in the present cases. However, some disadvantages may be present, such as relapse, TMD, and PCR when SSRO is performed in patients with a class II skeletal pattern [9-11]. In addition, the patients already had an open bite, and the maxillary segments moved upward with counter-clockwise rotation after osteotomies were performed. Moreover, they had AOB, which was one of the crucial risk factors for PCR [11]. These factors implied that the degree of mandibular counter- clockwise rotation would be larger, resulting in increased risk for the aforementioned complications.

To avoid the complications associated with SSRO for skeletal class II cases with $\mathrm{AOB}$ and a gummy smile, the present patients were followed with PPS. This postoperative management provided good skeletal and dental stability in skeletal class III patients based on our previous studies $[14,15]$. Segmental fixation after mandibular osteotomy was not performed in PPS. Therefore, inappropriate plate bending and seating of the proximal segment, which are the main factors for post-surgical relapse or TMD, can be omitted. Subsequently, the proximal segment must move to the physiologically ideal position for the newly created occlusion and jaw movement. In fact, there were no disadvantages associated with the temporomandibular joint, such as TMD, PCR, or limitation of mouth opening, after surgery in the present cases. Skeletal stability was acquired almost within 3 months after surgery in Case1. However, slight relapse of the mandible was observed in Case-2, although postsurgical orthodontic treatment was able to manage this. It was considered that elastic traction using a SAD was insufficient in Case-2. According to these outcomes, it was suggested that elastic traction with a SAD should be maintained for more than 10 weeks to resist the force of mouth-opening muscles, when the mandible moves anteriorly with counter-clockwise rotation after surgery in skeletal class II cases with $\mathrm{AOB}$ and a gummy smile.

According to the outcomes of our two cases, skeletal stability was almost achieved 3 months after surgery in skeletal class II patients with AOBs and gummy smiles who underwent maxilla-mandibular osteotomies with PPS. Furthermore, if the maxillary segment is moved with counter-clockwise rotation, it is considered that Le Fort I osteotomy with a horseshoe osteotomy may induce a better treatment outcome when compared with Le Fort I osteotomy alone. Of course, further research is required to conclude whether a combination of Le Fort I and horseshoe osteotomies or a sole Le Fort I osteotomy is better for acquiring long-term skeletal stability.

According to Figures 3 and 6, and Tables 1 and 2, the proximal segment 12 months after surgery swung anteriorly by approximately $10^{\circ}$ from the original position. Because no TMDs were observed and good outcomes were acquired in the present cases, the proximal segments were considered to be in physiologically ideal positions 12 months after surgery. This implies that the original position of the proximal segment is not always physiologically ideal, or can be far from the physiological position for a newly created occlusion after surgery in skeletal class II cases with $\mathrm{AOB}$ and a gummy smile. Interestingly, our previous study showed that the original position of the proximal segment was almost the same as the postoperative position of the proximal segment in class III cases [15]. It is considered that the proximal segment in skeletal class II and class III cases may be different before surgery and this difference may be one of the factors supporting a tendency for relapse in skeletal class II cases. The centric relation (CR) splint is sometimes applied for patient before surgery to acquire the preoperative physiological position of the condyle. There were no symptoms of TMD in both cases. Additionally, the postoperative ideally condylar position may not be same as preoperative condylar position in the concept of PPS. Moreover, the segments are not fixed each other after mandibular osteotomy. Thus the condylar position should be changed immediately right after the ramus splitting. Therefore, even if the condyle was seated at the physiological position before surgery, that does not make the sense. According to above mentioned reason, CR splint was not used before surgery in both cases. 
Citation: Ohba S, Yoshimura H, Matsuura T, Asahina I, Sano K (2016) Bimaxillary Osteotomy Using a Physiological Positioning Strategy for Skeletal Class II with Anterior Open Bite and Gummy Smile: Case Reports and Review of the Literature. Dentistry 6: 356. doi:10.4172/21611122.1000356

Page 6 of 6

\section{Conclusion}

According to the outcomes of the present cases, physiological positioning strategy may provide a reliable outcome when the maxilla is moved upward with counter-clockwise rotation and the mandible is moved anteriorly with counter-clockwise rotation in skeletal class II cases with $\mathrm{AOB}$ and a gummy smile. Further studies are needed to confirm, particularly, the position of the proximal segments before and after surgery.

\section{References}

1. Espeland L, Dowling PA, Mobarak KA, Stenvik A (2008) Three-year stability of open-bite correction by 1-piece maxillary osteotomy. Am J Orthod Dentofacial Orthop 134: 60-66.

2. Bosio JA, Justus $R$ (2013) Treatment and retreatment of a patient with a severe anterior open bite. Am J Orthod Dentofacial Orthop 144: 594-606.

3. Denison TF, Kokich VG, Shapiro PA (1989) Stability of maxillary surgery in openbite versus nonopenbite malocclusions. Angle Orthod 59: 5-10.

4. Fontes AM, Joondeph DR, Bloomquist DS, Greenlee GM, Wallen TR, et al. (2012) Long-term stability of anterior open-bite closure with bilateral sagittal split osteotomy. Am J Orthod Dentofacial Orthop 142: 792-800.

5. Abraham J, Bagchi P, Gupta S, Gupta H, Autar R (2012) Combined orthodontic and surgical correction of adult skeletal class II with hyperdivergent jaws. Nat $J$ Maxillofac Surg 3: 65-69.

6. Brammer J, Finn R, Bell WH, Sinn D, Reisch J, et al. (1980) Stability after bimaxillary surgery to correct vertical maxillary excess and mandibular deficiency. J Oral Surg 38: 664-670.

7. Proffit WR, Turvey TA, Phillips C (2007) The hierarchy of stability and predictability in orthognathic surgery with rigid fixation: an update extension. Head Face Med 3: 21.

8. Chen S, Lei J, Wang X, Fu XY, Farzard P, Yi B (2013) Short- and long-term changes of condylar position after bilateral sagittal split ramus osteotomy for mandibular advancement with Le Fort I osteotomy evaluated by cone-beam computed tomography. J Oral Maxillofac Surg 71: 1956-1966.

9. Cascone P, di Paolo C, Leonardi R, Pedullà E (2008) Temporomandibular disorders and orthognathic surgery. J Craniofac Surg 19: 687-692.

10. Wolford LM, Reiche-Fischel O, Mehra P (2013) Changes in temporomandibular joint dysfunction after orthognathic surgery. J Oral Maxillofac Surg 61: 655-660.

11. Hoppenreijs TJM, Maal T, Xi T (2013) Evaluation of condylar resorption before and after orthognathic surgery. Semin Orthod 19: 106-115.

12. Ohba S, Tasaki H, Tobita T, Minamizato T, Kawasaki T, et al. (2013) Assessment of skeletal stability of intraoral vertical ramus osteotomy with one-day maxilla- mandibular fixation followed by early jaw exercise. J Craniomaxillofac Surg 41 586-592.

13. Harada $K$, Okada $Y$, Nagura $H$, Enomoto $S$ (1994) A new repositioning system for the proximal segment in sagittal split ramus osteotomy of the mandible. Int J Oral Maxillofac Surg 23: 71-73.

14. Ohba S, Nakao N, Nakatani Y, Kawasaki T, Minamizato T, et al. (2014) The skeletal stability after maxillo-mandibular osteotomy with a "physiological positioning strategy". Br J Oral Maxillofac Surg 52: 965-969.

15. Ohba S, Yoshida M, Kohara H, Kawasaki T, Minamizato T, et al. (2014) Short lingual osteotomy without fixation: a new strategy for mandibular osteotomy known as "physiological positioning". Br J Oral maxillofac Surg 52: e9-e13.

16. Harada K, Sumida E, Enomoto S, Omura K (2002) Post-operative stability of the maxilla treated with Le Fort $I$ and horseshoe osteotomies in bimaxillary surgery. Eur J Orthodont 24: 471-476.

17. Bell WH, McBride KL (1977) Correction of the long face syndrome by Le Fort I results. A report on some new technical modifications and treatment results. Oral Surg Oral Med Oral Path 44: 493-520.

18. Wehrbein H, Bauer W, Diedrich P (1996) Mandibular incisors, alveolar bone and symphysis after orthodontic treatment a retrospective study. Am J Orthod Dentofacial Orthop 110: 239-246.

19. Weltman B, Vig KW, Fields HW, Shanker S, Kaizar EE (2010) Root resorption associated with orthodontic tooth movement: a systematic review. Am J Orthod Dentofacial Orthop 137: 462-476.

20. Tsui WK, Chua HD, Cheung LK (2012) Bone anchor systems for orthodontic application: a systematic review. Int J Oral Maxillofac Surg 41: 1427-1438.

21. Ahmed VKS, Rooban T, Krishnaswamy NR, Mani K, Kalladka G (2012) Root damage and repair in patients with temporary skeletal anchorage devices. Am J Orthod Dentofacial Orthop 141: 547-555.

22. Kaku M, Kojima S, Sumi H, Koseki H, Abedini S, et al. (2012) Gummy smile and facial profile correction using miniscrew anchorage. Angle Orthod 82: 170-177.

23. Nishimura M, Sannohe M, Nagasaka H, Igarashi K, Sugawara J (2014) Nonextraction treatment with temporary skeletal anchorage devices to correct a Class II Division 2 malocclusion with excessive gingival display. Am J Orthod Dentofacial Orthop 145: 85-94.

24. Capelozza Filho L, Cardodo Mde A, Reis SA, Mazzottini R (2006) Surgicalorthodontic correction of long-face syndrome. J Clin Orthod 40: 323-332.

25. Ribeiro GL, Regis S Jr, da Cunha Tde M, Sabatoski MA, Guariza-Filho O, et al. (2010) Multiloop edgewise archwire in the treatment of a patient with an anterior open bite and a long face. Am J Orthod Dentofacial Orthop 138: 89-95.

26. Shimo T, Nishiyama A, Jinno T, Sasaki A (2013) Severe gummy smile with class II malocclusion treated with LeFort I osteotomy combined with horseshoe osteotomy and intraoral vertical ramus osteotomy. Acta Med Okayama 67: 5560 . 